\title{
Trop2 as an oncogene in gastric cancer by regulating the PI3K/Akt signaling pathway
}

\author{
Wei Zhao ${ }^{1,2 \Delta}$, Qi Tang ${ }^{3 \Delta}$, Xingwang Kuai ${ }^{1}$, Xiaochen Huang ${ }^{1}$, Yaru Xu', Tingting Yang ${ }^{1}$, Yuan Chen ${ }^{3}$, \\ Mingjiong Zhang ${ }^{3}$, Zhenning Qiu ${ }^{3}$, Jin Zhu ${ }^{3,4}$, Wenbin Huang ${ }^{2^{*}}$, Zhenqing Feng ${ }^{1,3^{*}}$ \\ ${ }^{1}$ Department of Pathology, Nanjing Medical University, Nanjing, Jiangsu, 211166, China; \\ ${ }^{2}$ Department of Pathology, the First Hospital Affiliated to Nanjing Medical University, Nanjing, Jiangsu, 210029, China; \\ ${ }^{3}$ Key Laboratory of Antibody Technique of Ministry of Health, Nanjing Medical University, Nanjing, Jiangsu, 211166, China; \\ ${ }^{4}$ Huadong Medical Institute of Biotechniques, Nanjing, Jiangsu, 210002, China
}

\begin{abstract}
High Trop2 expression relates to aggressive tumor behavior and contributes to poor overall survival rates in gastric cancer (GC) patients. However, little is known about the molecular mechanism of Trop2 in the carcinogenesis of GC. We found that over-expressed Trop2 induced cell proliferation and clone formation, inhibited cell apoptosis and induced S cell cycle arrest in GC cell lines, meanwhile, knockdown Trop2 inhibited cell proliferation and clone formation, induced cell apoptosis and inhibits S cell cycle arrest in vitro. Moreover, Trop2 depletion inhibited tumor growth, the anti-tumor rate in this report being $22.53 \%$ in vivo. In addition, Trop2 activated the PI3K/Akt signaling pathway to promote GC malignant progression. These results indicated that Trop2 is a critical regulation factor in the progression of GC, which may help to lead a novel insight into understanding the mechanism of the Trop2 in the pathogenesis of GC.
\end{abstract}

Keywords: Trop2, gastric cancer, signaling pathway

\section{INTRODUCTION}

Gastric cancer (GC) is the fourth common cancer and the second leading cancer-related cause of death worldwide $^{[1-3]}$. Despite GC declining in incidence in Western countries, gastric carcinoma is a major cause of death in numerous parts of the world, especially in East Asia ${ }^{[4]}$. Fifty-eight percent of global GC deaths occur in China, Korea, and Japan ${ }^{[5]}$. Although the early detection rate of GC has increased, there are still many patients suffering from distant metastasis. Thus, it is important to identify oncogenes that promote gas-

\footnotetext{
*Correspondence to: Zhenqing Feng, E-mail: fengzhenqing@njmu. edu.cn; Wenbing Huang, E-mail: wbhuang348912@126.com.

${ }^{\Delta}$ These authors contributed equally to this work.
}

tric cancer metastasis. These oncogenes may not only serve as biomarkers for gastric cancer progression, but also as molecular targets for gastric cancer therapy.

The human oncogene, trophoblast cell surface glycoprotein (TACSTD2/Trop2) has been given particular attention in our lab since 2009. Trop2, located at chromosome 1q32, was first identified on the surface of human trophoblast cells ${ }^{[6-8]}$. Our early experimental results indicated highly expressed Trop2 in many solid tumors, moreover its expression relateed to aggressive tumor behavior $^{[9-11]}$. Subsequently we prepared several anti-Trop2 antibodies, including human anti-Trop2 antibody IgG, fragment Fab and so on, these antibodies have been found able to bind to specific Trop2 antigen and inhibit the proliferation, migration and invasion of the tumor cells in vivo and in vitro.

The phosphoinositide 3-kinase (PI3K) signal- 
ing pathway represents the most important signaling pathway relating to cell proliferation, apoptosis, metabolism and is often activated in $\mathrm{GC}^{[12,13]}$. PI3K catalyzes the production of phosphatidylinositol3,4,5-trisphosphate(PIP3) by phosphorylating phosphatidylinositol (PI), phosphatidylinositol4-phosphate (PIP) and phosphatidylinositol-4,5bisphosphate (PIP2). Growth factors and hormones trigger this phosphorylation event, which in turn coordinates cell growth, cell cycle entry, cell migration, and cell survival ${ }^{[14]}$. PI3K is a lipid kinase containing two subunits, catalytic (p110) and regulatory (p85) ${ }^{[15-17]}$. Akt is activated by phospholipid binding and activation loop phosphorylation within the carboxy terminus at Ser473 by $\mathrm{PDK}^{[18]}$. Akt promotes cell survival by inhibiting apoptosis, and moreover phosphorylating and inactivating several targets, including c-Raf. PTEN phosphatase is a major negative regulator of the PI3K/Akt signaling pathway ${ }^{[19]}$.

In the present study, we aimed to investigate the expression and role of Trop2 in vivo and in vitro and further clarify the role of Trop2 through activating the PI3K/Akt signaling pathway in GC cell lines.

\section{MATERIALS AND METHODS}

\section{Cell culture, reagents and transfections}

Seven human GC cell lines (MKN45, MKN28, MGC803, SGC7901, HGC27 and BGC823) and a normal human gastric epithelial cell lines (GES-1) were purchased from KeyGEN BioTECH (Nanjing, China). Cells were cultured at $37^{\circ} \mathrm{C}$ in a $5 \% \mathrm{CO}_{2}$ humidified atmosphere in RPMI-1640 containing 10\% fetal bovine serum(FBS). Trop2 were listed in Supplementary Table S1. Transfection into GC cells was carried out using Lipofectamine 2000 transfection reagent (Invitrogen, Shanghai, China) according to the manufacturer's instructions. The efficiency of overexpression or Rnock-down were listed in Supplementary Figure S1-S2.

\section{RNA extraction, reverse transcription, and quantitative PCR}

Cell RNA was extracted by TRIzol reagent (Invitrogen, Carlsbad, CA, USA) and reverse transcribed into cDNA using a PrimeScript ${ }^{\mathrm{TM}} \mathrm{RT}$ reagent kit (TaKaRa, Dalian, China) in accordance with the manufacturer's instructions. The primers of Trop2 (Allin-One qPCR Primer) were purchased from GeneCopoeia (Guangzhou, China), GAPDH (internal control) were purchased from Genscript (Nanjing, China). qRT-PCR was performed on an ABIPRISM 7500HT
Sequence Detection System (Applied Biosystems, Foster City, CA, USA) in 96-well plates. Relative expression levels were calculated as ratios normalized against those of GAPDH. Results were normalized to respective internal controls. The $\mathrm{C}_{\mathrm{T}}-$ value for each sample was calculated using the $\Delta \Delta \mathrm{C}_{\mathrm{T}}$ method, and results were expressed as $2^{-\Delta \Delta \mathrm{C}_{\mathrm{T}}}$.

\section{Western blot analysis}

Cells were lysed using standard methods. Cellular proteins were separated by $10 \%$ SDS-PAGE and transferred onto nitrocellulose membranes. The membranes were blocked with TBS containing $0.1 \%$ Triton X-100 and 5\% nonfat milk powder for 2 hours at room temperature(RT), then were incubated with anti-Trop2 (R\&D Systems, Minneapolis, USA), antiPTEN(CST, USA), anti-PI3K(CST, USA), antiAkt(CST, USA), anti-p-Akt(CST, USA), anti-p-cRaf(CST, USA), anti-GAPDH (CST, USA) antibodies at $4{ }^{\circ} \mathrm{C}$ overnight. After being washed, the membranes were incubated with HRP-conjugated anti-IgG at RT for 1 hour. Signal detection was carried out with an ECL system (Tanon, Shanghai, China).

\section{CCK-8 assay}

Cells at a number of $5 \times 10^{3}$ per well were seeded in the 96-well plate and incubated. Cell growth viability was measured with a Cell Counting Kit-8 (Beyotime, Shanghai, China) following the manufacturer's instructions. Absorbance was then recorded at $450 \mathrm{~nm}$ using Elx800 Reader (BioTECH Instruments Inc., Winooski, VT, USA).

\section{Colony formation assay}

The transfected GC cell lines were placed in a fresh 6 -well plate and maintained in containing 10\% FBS. After 24 hours, the medium was replaced with new medium containing $\mathrm{G} 418(300 \mathrm{mg} / \mathrm{mL})$ or puro $(10$ $\mu \mathrm{g} / \mathrm{mL}$ ). After 14 days, cells were fixed with methanol and stained with $0.1 \%$ crystal violet. Visible colonies were manually counted.

\section{Cell cycle and cell apoptosis analyzed by flow cytometry}

the trypsinized cells $\left(1 \times 10^{6}\right)$ were fixed in $70 \%$ ethanol at $-20{ }^{\circ} \mathrm{C}$ for $24 \mathrm{~h}$. The fixed cells were then washed with PBS, and incubated with RNaseA (0.25 $\mathrm{mg} / \mathrm{mL}$ ) for $30 \mathrm{~min}$ at $37^{\circ} \mathrm{C}$, and $5 \mu \mathrm{L}$ of propidium iodide (PI, KeyGen, Nanjing, China) was then added to the cell suspension; Cells were washed with PBS and incubated with $5 \mu \mathrm{L}$ Annexin V-APC-7AAD and $10 \mu \mathrm{L}$ PI for $5 \mathrm{~min}$ at $37^{\circ} \mathrm{C}$. The mixture was incubated at RT for $30 \mathrm{~min}$ in the dark. The suspended 
cells were analyzed for cell cycle using the FACS Calibur Flow Cytometer (BD Biosciences, San Jose, CA, USA).

\section{Animal experiments}

Animal experiments were performed with the approval of the Institutional Committee for Animal Research and in conformity with national guidelines for the care and use of laboratory animals. ShRNA-5, shRNA-NC transfected BGC823 cells $\left(1 \times 10^{6}\right.$ cells in $100 \mu \mathrm{L}$ ) were injected subcutaneously into the flanks of each 4-week-old immunodeficiency nude mouse. BCG823-shRNA-NC were injected subcutaneously into the left flank and BCG823-shRNATrop2 were injected subcutaneously into the right flank. Tumor growth was evaluated weekly for at least 5 weeks. Then the mice were sacrificed, necropsies were performed, and tumors were evaluated. Tumor volumes were calculated by the following formula: $V$ $=\pi A B^{2} / 6$, where $A$ is the largest diameter, and $B$ is the perpendicular diameter. The tumor tissues were used to perform immuno-staining analysis of Trop2 protein expression.

\section{Statistical analysis}

The SPSS18.0 statistical software package (SPSS Inc., Chicago, IL, USA) was used for general statistical analysis. The differences between groups were estimated by Values of $P$ less than 0.05 were considered statistically significant.

\section{RESULTS}

\section{Over-expression of Trop2 induces cell prolif-}

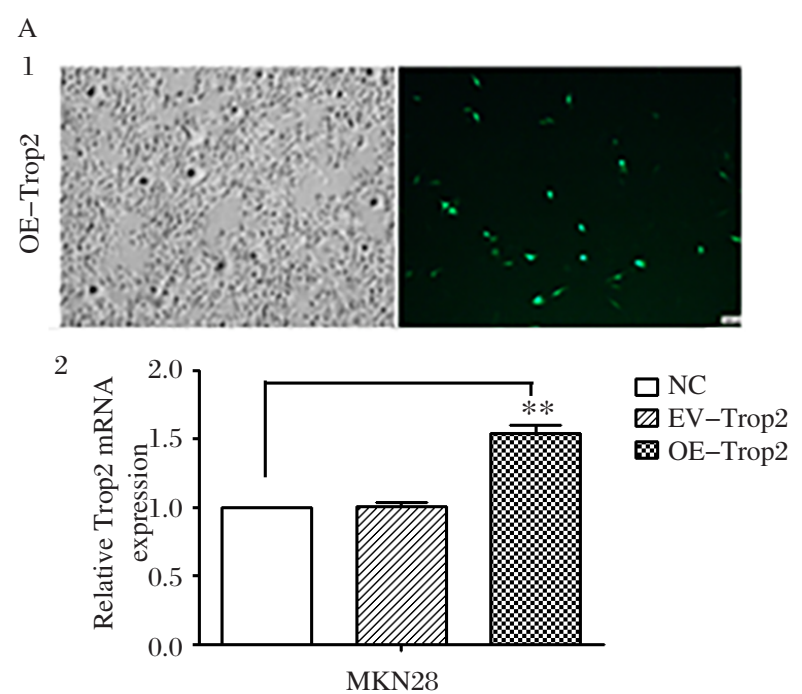

eration and clone formation, inhibits cell apoptosis and induces $S$ cell cycle arrest in GC cell lines

The expression of Trop2 among 6 types of GC cell lines (MGC803, BGC823, MKN45, MKN28, HGC27, SGC7901) and a normal gastric epithelial cell line (GES-1) were analyzed by qRT-PCR (Fig. 1A). We found that Trop2 was relatively high-expressed in MKN45 and BGC823 and showed a relatively low-expression in MKN28 and MGC803. So these four types of GC cell line were used in the following studies.

First, we constructed a plasmid vector, which overexpressed Trop2, named OE-Trop2. The expression efficiency of Trop2 was $1.56 \pm 0.06$ fold higher than the normal control (NC) and the cloning vector (VETrop2) in MKN28, and was 3.86 \pm 0.39 fold higher than NC and VE-Trop2 in MGC803 (Supplementary Fig. S1).

Then, CCK8 assay was used to assess the cell proliferation 24, 48 and 72 hours after OE-Trop2 plasmid transfection. Compared with NC and VE-Trop2, a significant increase of cell proliferation was detected in MKN28 at $72 \mathrm{~h}$ and in MGC803 at 48 and $72 \mathrm{~h}$ after being transfected with OE-Trop2. But no difference was observed between NC and VE-Trop2 group (Fig. 1B). Colony formation assay was performed to further examine the effect of OE-Trop2 on the proliferation of GC cell lines. As shown in Fig. 1C, the cell colony numbers of MKN28 and MGC803 transfected with OE-Trop2 were significantly higher than those transfected with VE-Trop2. Therefore, the results of colony formation assay, consistent with those of

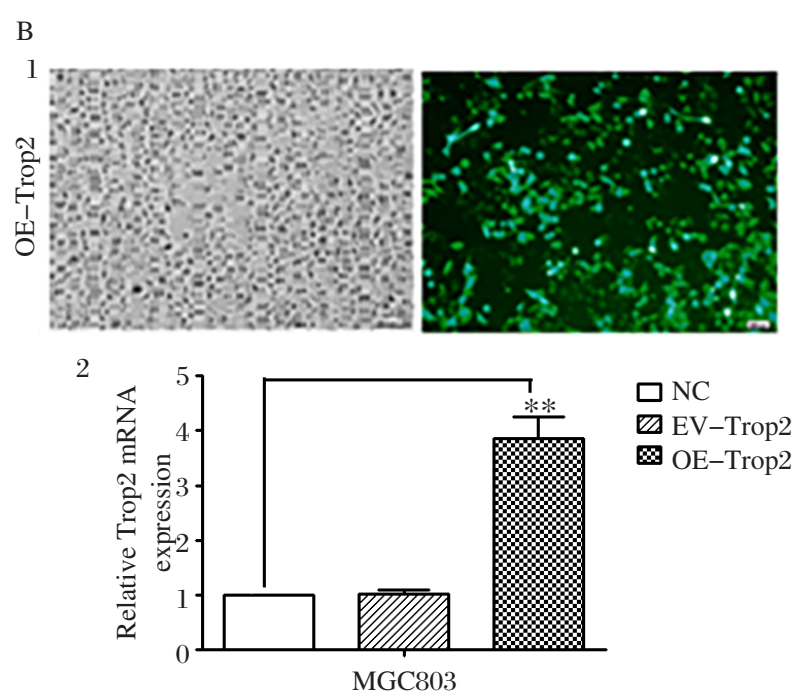

Fig. S1. The OE-Trop2 plasmid transfection efficiency of gastric cancer cells were analyzed by fluorescent and qRT-PCR. (A1):MKN28 OE-Trop2 by fluorescent; (A2): MKN28 OE-Trop2 byqRT-PCR; (B1): MGC803 OE-Trop2 by fluorescent; (B2):MGC803 OETrop2by qRT-PCR 

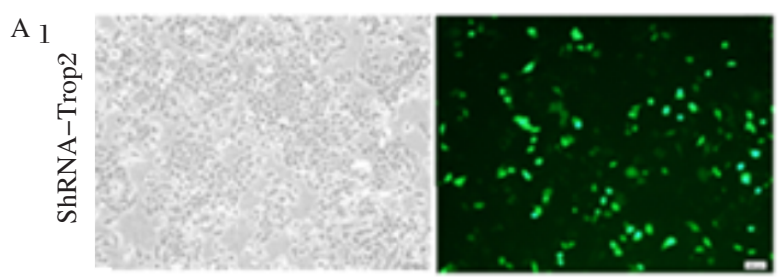

2

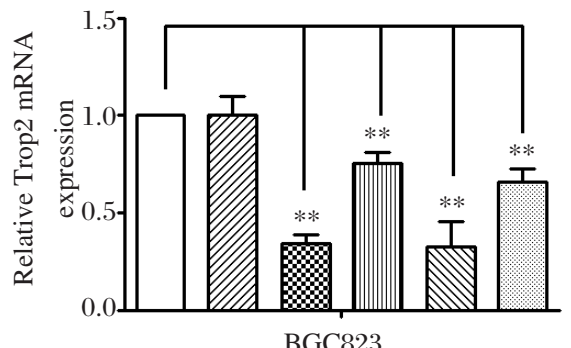

$\mathrm{B}_{1}$

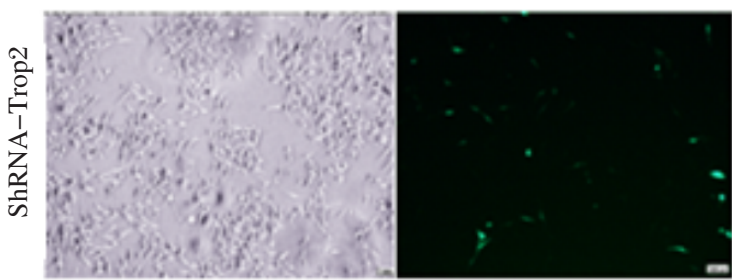

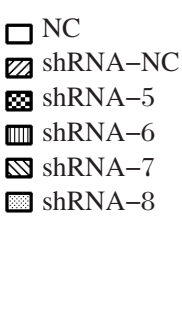

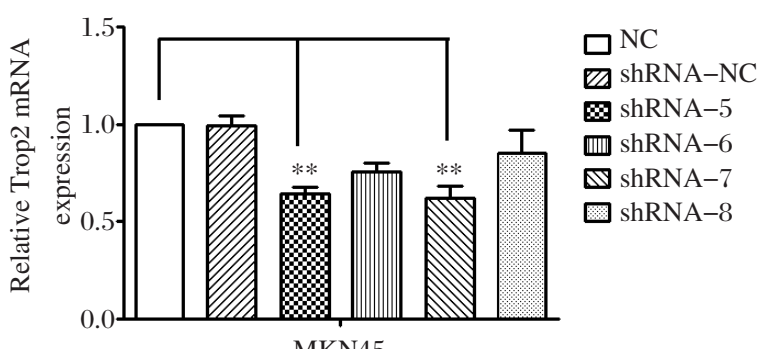

MKN45

Fig. S2. The ShRNA-Trop2 plasmid transfection efficiency of gastric cancer cells were analyzed by fluorescent and qRT-PCR. (A1):BGC823 ShRNA-Trop2 by fluorescent; (A2): BGC823 ShRNA-Trop2 byqRT-PCR; (B1): MKN45 ShRNA-Trop2 by fluorescent; (B2): MKN45 ShRNA-Trop2by qRT-PCR

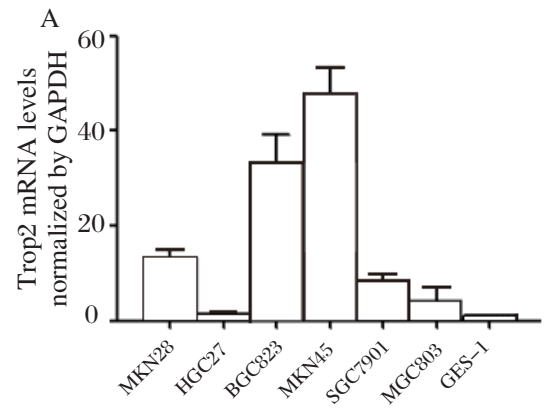

C

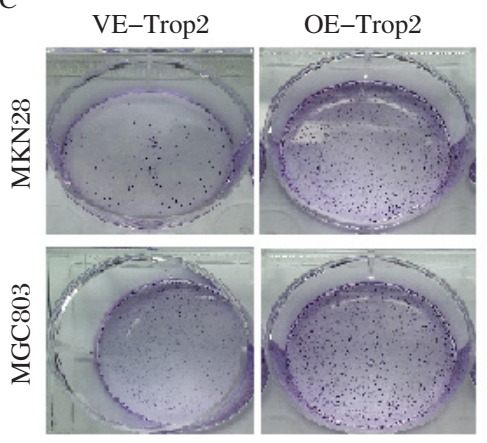

B

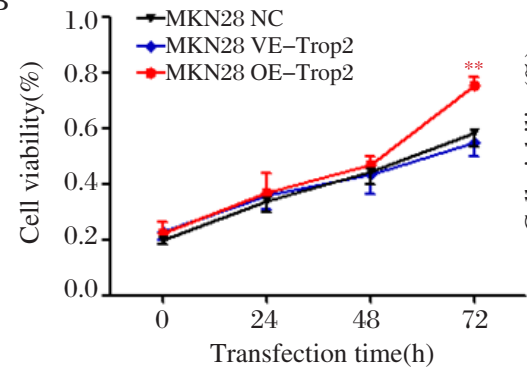

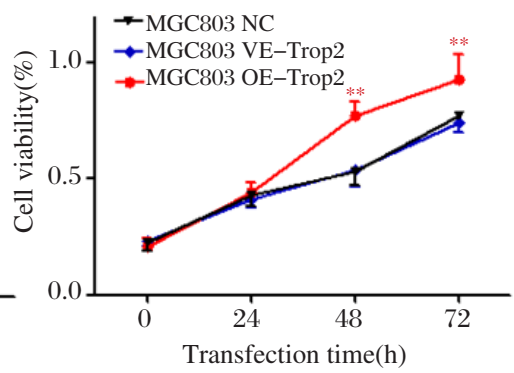

D
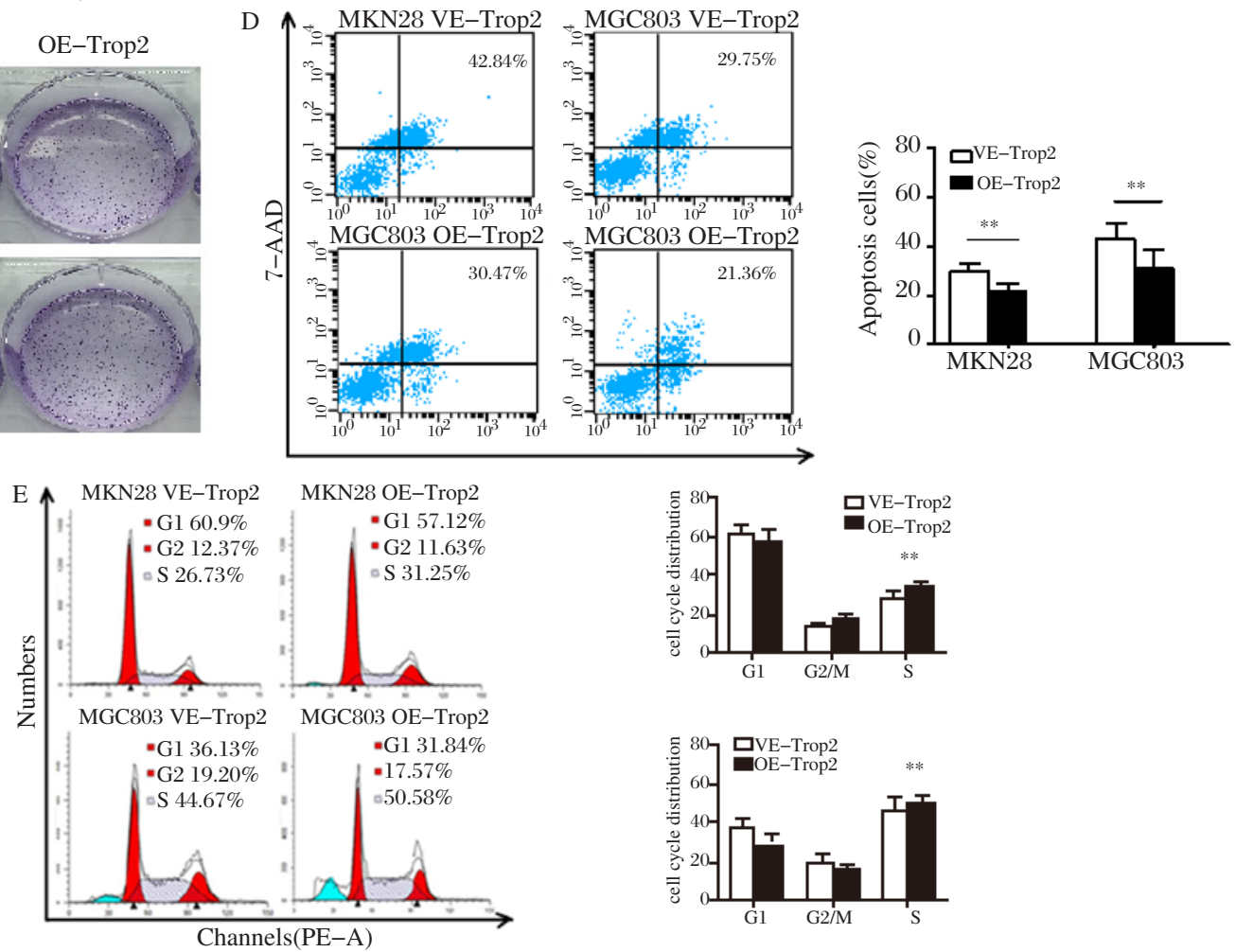

Fig. 1. Trop2 over-expression induces cell proliferation and clone formation, inhibits cell apoptosis and induces $\mathrm{S}$ cell cycle arrest in GC cell lines. A: the statistical results of Trop2 expression levels in different gastric cancer cell lines and a normal gastric epithelial cell line (GES-1) by qRT-PCR, and GAPDH was treated as internal control; B: the proliferation ratio of OE-Trop2 transfected MKN28 and MGC803 cells tested by CCK8 at 0, 24, 48, $72 \mathrm{~h}$ time point; C: colony formation of OE-Trop2 transfected MKN28 and MGC803 cells; D: the apoptosis cells ratio of OE-Trop2 transfected MKN28 and MGC803 cells analyzed by FACS. E: the cell cycle of OE-Trop2 transfected MKN28 and MGC803 cells analyzed by FACS. 
CCK8 assay, suggest that Trop2 can induce the proliferation of GC cells.

Cell cycles of MKN28 and MGC803 transfected with OE-Trop2 or VE-Trop2 were examined using FACS. MKN28 and MGC803 transfected with OE-Trop 2 had more cells (nearly $31.25 \%$ and $50.58 \%$ ) arrested in $\mathrm{S}$ phase $72 \mathrm{~h}$ after transfection compared with VE-Trop2 transfected cells. These results suggested that the $\mathrm{S}$ cell cycle progression was induced after the over-expression of Trop2 (Fig. 1E).

All above indicated that Trop2 has oncogenic properties and OE-Trop2 could increase cell proliferation and clone formation, inhibit cell apoptosis and induce $\mathrm{S}$ cell cycle arrest in GC cell lines.

\section{Knockdown of Trop2 inhibits cell proliferation and clone formation, induces cell apoptosis and G1 cell cycle arrest in GC cell lines}

MKN45 and BGC823 were used in subsequent knockdown experiments for their high expression of Trop2. Different vectors (shRNA-5, -6, -7, -8) expressing short hairpin RNAs (shRNA) against Trop2 were tested. The most effective down-regulation of Trop2 was achieved with two sequences: shRNA-5 (65\% in BGC823, 35\% in MKN45), and shRNA-7 (67\% in BGC823, 37\% in MKN45) (Supplementary Fig. S2). As a control, cells were transduced with control scrambled shRNA vectors(shRNA-NC).

We examined the cell proliferation at 24,48 , and 72 hours after transfection of shRNA-Trop2 used CCK8 assay. Compared with the non-transfected group (NC) and control scrambled shRNA vectors transfected group (shRNA-NC), a significant decrease of cell proliferation was detected in MKN45 at $48 \mathrm{~h}$ and BGC823 at 48 and $72 \mathrm{~h}$ after being transfected with shRNA-5 and shRNA-7 plasmid. Meanwhile, no difference was observed between the NC and shRNANC group (Fig. 2A).

Colony formation assay showed that the cell colony numbers of MKN45 and BGC823 transfected with shRNA-5 and shRNA-7 were significantly lower than those transfected with shRNA-NC (Fig. $2 \boldsymbol{B}$ ). These results along with the results of CCK8 assay further indicated that down-regulation of Trop2 could inhibit the proliferation of GC cells in vitro.

We next testified the apoptosis cells ratio and the distribution of cell cycles using FACS. The apoptosis ratios of MKN45 transfected with shRNA-5 and shRNA-7 were obviously higher than those transfected with shRNA-NC, and the similar results were also observed in BGC823(Fig. 2C). In comparison with shRNA-NC group, the shRNA-5 transfected
MKN45 group showed cell cycle arrest in Gl phase 72 hours after transfection, characterized by the presence of nearly $68 \%$ of cells in the Gl phase, the presence of about $20 \%$ of cells in S phase. While in BGC823 cell lines, the shRNA-5 transfected group showed cell cycle arrest in G2/M phase 72 hours after transfection, characterized by the presence of nearly $20 \%$ of cells in the G2/M phase, the presence of about $30 \%$ of cells in $\mathrm{S}$ phase. The results indicated that the down-regulation of Trop2 inhibited S cell cycle arrest (Fig. 2D).

These results indicated that knockdown of Trop2 expression could inhibit cell proliferation and clone formation, induces cell apoptosis and Gl cell cycle arrest in GC cell lines.

\section{Trop2 depletion inhibits tumor growth in vivo}

Xenograft model was used to evaluate the effects of shRNA-Trop2 on the growth of the GC cells in vivo, in which the BGC823 cells treated with shRNA-5 or shRNA-NC $\left(1 \times 10^{6}\right.$ cells/mouse $)$ were subcutaneously injected into the flanks of immunodeficient nude mice for the developing of tumors. The mice were sacrificed after 5 weeks and the tumors were examined (Fig. 3B). There was no animal dead during the experiment. During the whole tumor growth process, tumors from the shRNA-5 transfected BGC823 cells grew slower than those of shRNA-NC transfected ones (Fig. 3C). After 5-week's inocula-

OE-Trop2 plasmid profile
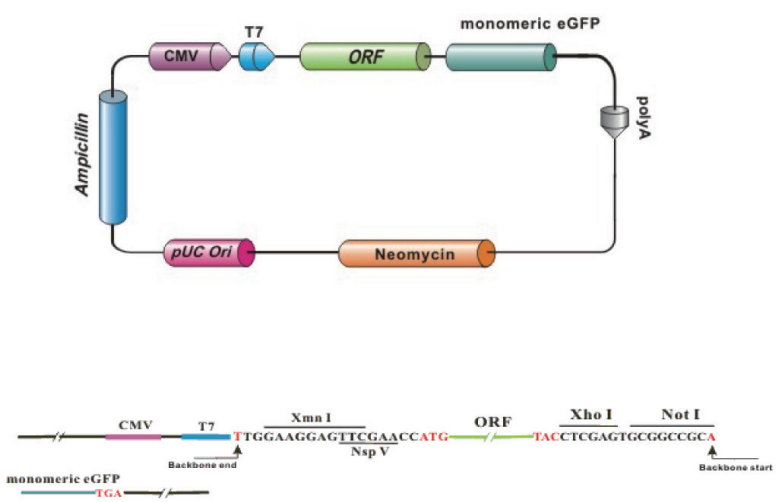

ShRNA-Trop2 plasmid profile

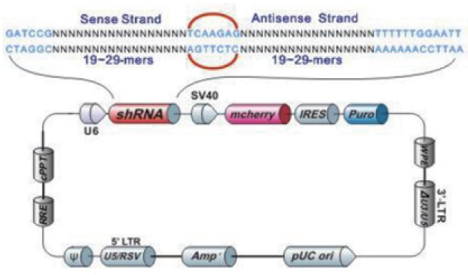

ShRNA Target Sequences

Clone Name Symbol Location Length Target Sequence HSH010940-5-LVRU6MP (OS398373) TACSTD2 443 HSH 010940-6- LVRU6MP (OS398374) TACSTD2 HSH010940-7- LVRU6MP (OS398375) TACSTD2 HSH010940-8- LVRU6MP (OS398376) TACSTD2

$\begin{array}{lll}443 & 21 & \text { gtgtcccaccaacaagatgac } \\ 1097 & 21 & \text { ggagcgcacgctcatctatta } \\ 835 & 21 & \text { cettcaaccactcagacctgg } \\ 549 & 21 & \text { tccaagtgtctgctgctcaag }\end{array}$



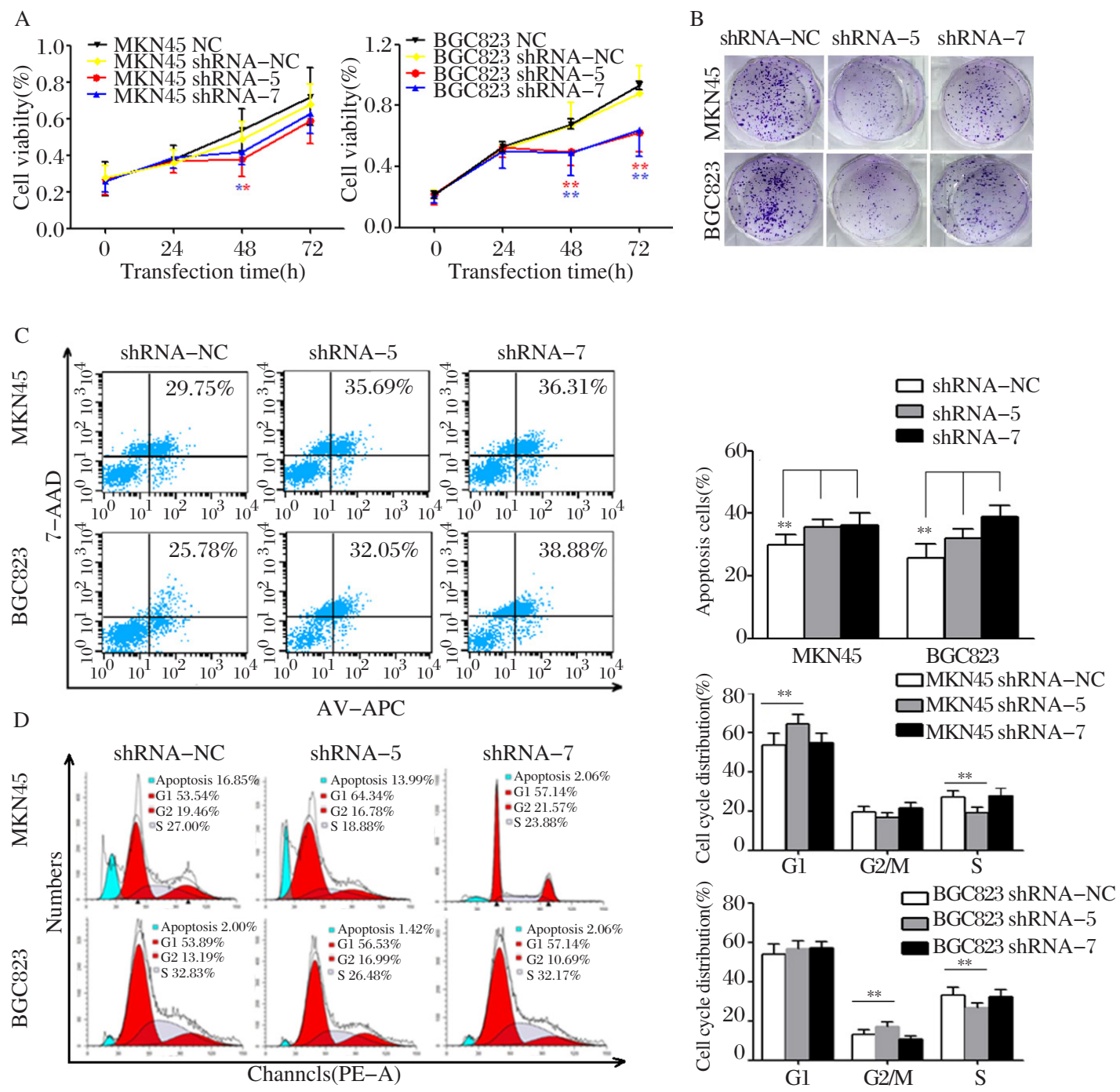

Fig. 2. Trop2 knockdown inhibits cell proliferation and clone formation, induces cell apoptosis and G1 cell cycle arrest in GC cell lines. A: the proliferation ratio of shRNA-5 and shRNA-7 transfected MKN45 and BGC823 cells tested by CCK8 at 0, 24, 48, 72 h time point; B: colony formation of shRNA-5 and shRNA-7 transfected MKN45 and BGC823 cells; C: the apoptosis cells ratio of shRNA-5 and shRNA-7 transfected MKN45 and BGC823 cells analyzed by FACS; D: the cell cycle of shRNA-5 and shRNA-7 transfected MKN45 and BGC823 cells analyzed by FACS.

tion, the average weight of tumors developed from shRNA-5 transfected BGC823 cells [(204 \pm 116$) \mathrm{mg}$ ] was significantly smaller than those of control group $[(321 \pm 152) \mathrm{mg}, P=0.014]$, Immunostaining analysis of Trop2 protein was performed in resected tumor tissues. As shown in Fig. 3D, the expression level of Trop2 protein in tumors formed from shRNA-5 transfected BGC823 cells was significantly lower than those of the shRNA-NC group. These results suggest that down-regulated Trop2 expression could inhibit proliferation capacity of GC cells in vivo.

\section{Trop2 promotes GC malignant progression through PI3K/Akt signaling pathway}

Western blot analysis revealed that compared with the VE-Trop2 transfected groups, the expression levels of PI3K-p110 and p-Akt were markedly increased in the OE-Trop2 transfected MKN28 and MGC803 cells, while no difference was observed in Akt protein expression. Compared with the VE-Trop2 transfected groups, the protein expression levels of PTEN were markedly decreased in the OE-Trop2 transfected MKN28 and MGC803 cells. We found no significant difference on the expression levels of $\mathrm{p}-\mathrm{c}-\mathrm{Raf}$ between VE-Trop2 and OE-Trop2 transfected GC (MKN28 and MGC803) cells(Fig. 4). Next, we detected the activation of the PI3K/Akt signaling pathway after Trop2 was downregulated by shRNA-5 in GC (MKN45 and BGC823) cells by Western blot, and the results were consistent with those of the OE-Trop2 in GC cells. 
A

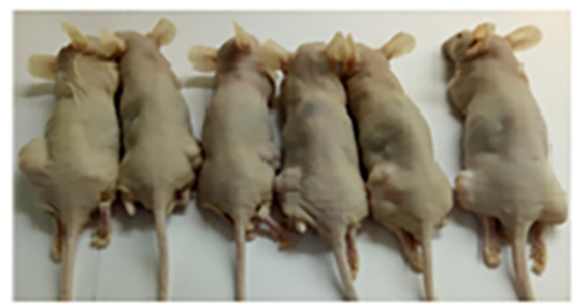

B

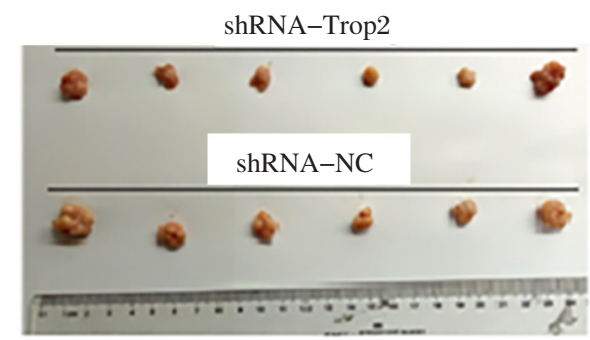

$\mathrm{C}$

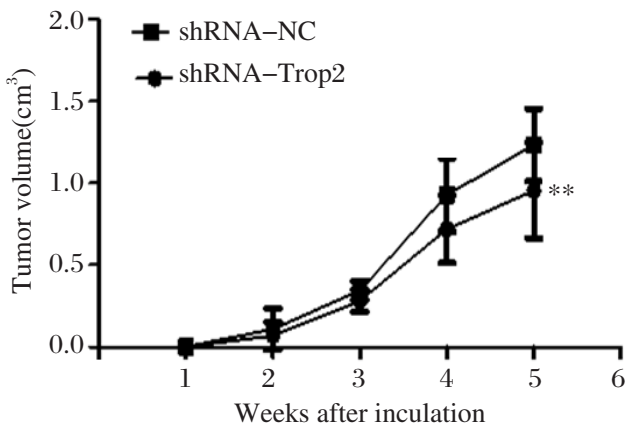

$\mathrm{D}$

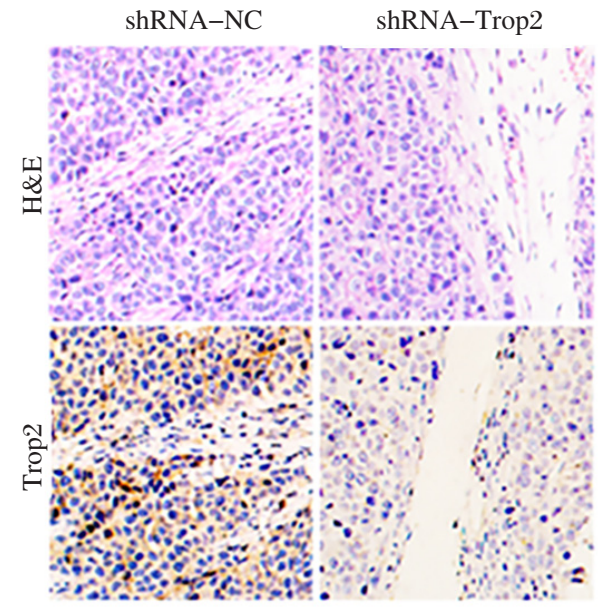

Fig. 3 Trop2 depletion inhibits tumor growth in vivo. A: Tumor formation in nude mice; B: Xenografts from nude mice 5 weeks after inoculation; $\mathrm{C}$ : Tumor volumes were examined every week after the nude mice were injected with BGC823 cells stably transfected with shRNA-NC or shRNA-5; D: Tumors developed from shRNA-Trop2 transfected GC cells showed a lower level of Trop2 protein expression than that of shRNANC transfected GC cells. Upper: H\&E staining; Lower: immunostaining $(\times 200)$. Data are shown as mean \pm SE. ${ }^{* *} P<0.01(\mathrm{n}=)$.

Thus, our present results indicate that Trop2 may facilitate GC malignant progression through the PI3K/ Akt signaling pathway.

\section{DISCUSSION}

GC is the most prevalent digestive system malig- nancy in the world, and China has more GC patients than any other country ${ }^{[20]}$. Our previous study showed that, Trop2, as an oncogene, highly expressed in GC tissues. The transmembrane protein Trop2 is extensively expressed in most cancers, but not all, and also has a differential expression in certain normal tissues. It has been reported that Trop2 is highly expressed in the following solid tumor cancers: colorectal cancer ${ }^{[2]]}$, breast cancer ${ }^{[22]}$, pancreatic cancer ${ }^{[23]}$, cervix cancer ${ }^{[24]}$, squamous cell carcinoma ${ }^{[25]}$, gastric cancer ${ }^{[20]}$, certain lung cancers ${ }^{[26]}$, prostate cancer ${ }^{[27]}$, bladder cancer $^{[28]}$, breast cancer ${ }^{[9]}$. However, it was down-regulated in non-small lung cancer ${ }^{[29]}$. Trop2 is also up-regulated in several hematologic malignancies: leukemia, extranodal nasal type lymphoma (ENK/TL), and nonHodgkin's lymphoma (NHL) ${ }^{[30]}$.

It has been reported that Trop2 mediates several signaling pathways ${ }^{[30]}$. High expression of Trop2 can activate MAPK signaling pathway, by increasing $\mathrm{Ca}^{2+[31]}$, and decreasing AP-1 and p27. But interestingly, Vidmar $\mathrm{T}^{[32]}$ reports that high expression of Trop2 inhibits the IGF-1R signaling pathway, and then attenuates lung cancer proliferation and malignancy. Trop2 is also reported to be able to bind $\beta$-catenin to drive stem cell self-renewal ${ }^{[33]}$.

In the present study, we demonstrated that over expression of Trop2 in GC cell lines (MKN28 and MGC803) could induce cell proliferation, and clone formation, inhibit cell apoptosis and induce $\mathrm{S}$ cycle arrest, and vice versa. These results are consistent with the findings of Lin $\mathrm{H}$ et al. ${ }^{[1]}$, who reported that Trop2 promotes the malignancy functions of ductal breast cancer cells. However, we also found two GC cell lines (MKN45 and MKN28) did not display similar results as for the other GC cell lines (BGC823 and MGC803) in the cell cycle distribution assays. This may partly due to the lower efficiency of plasmid transfection, although we improved several types of transfected ways. Furthermore, we found down regulation of Trop2 inhibited xenografted tumor's growth in vivo. This further confirmed the results in vitro.

In addition, we also found Trop2 facilitate GC malignant progression through the PI3K/Akt signaling pathway. Increasing evidences indicate that the PI3K/ Akt signaling pathway plays a vital role in the carcinogenesis and development of the malignant tumor, and its different downstream target genes are able to crosstalk with other pathways, therefore displaying a wide range of biological functions such as cell growth, cell cycle, apoptosis, migration and invasion, and so on. Our study shows the PI3K/Akt signaling pathway is likely to play an important anticancer role in various tumors, such as pancreatic cancer, rhabdomyosarcoma, 


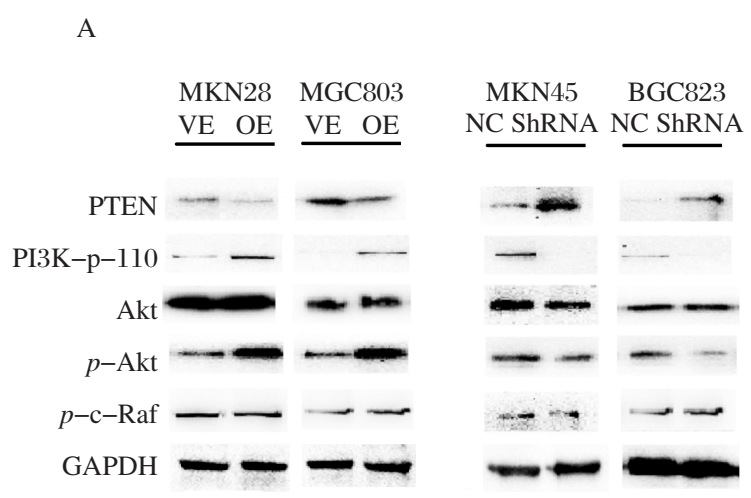

$\mathrm{B}$
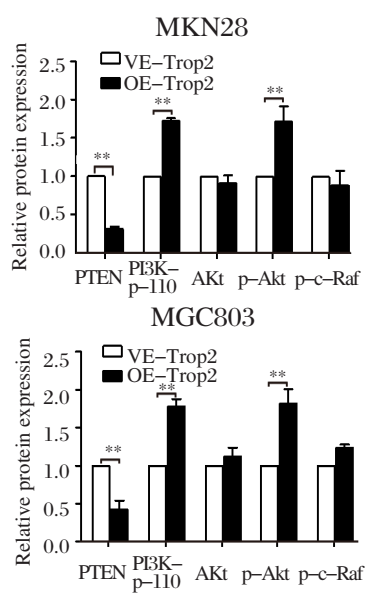

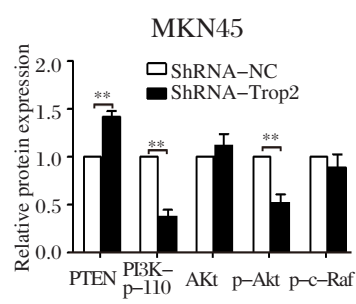

MGC823

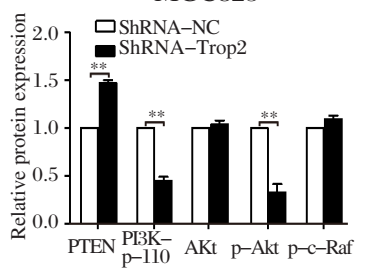

Fig. 4 Trop2 promotes GC malignant progression through PI3K/Akt signaling pathway.The representative Western blot results for PI3K/ Akt signaling pathway. The relative protein expression levels were obtained from three independent experiments, GAPDH was used as a control, mean \pm SD, ${ }^{* * *} P<0.01$.

gastric cancer and others ${ }^{[14]}$. In this study, we elucidate a novel Trop2-PI3K-Akt signaling pathway regulatory network in GC cells, and we believe our findings provide a new point for understanding the mechanism of Trop2 in the process of GC. Collectively, these results highlight that Trop2 is a critical regulation factor in the progression of GC. Nevertheless, these studies are currently limited, as the down-streaming target genes of PI3K/Akt have not as yet been studied satisfactorily, and there is a lack of clinical evidence to support the results. In order to fully investigate, future studies are required to this aim.

\section{Acknowledgments and funding}

This work was supported by grants from the $\mathrm{Na}-$ tional Natural Science Foundation of China (No. $81601618)$ and is the innovation of the graduate student training project of Jiangsu province (No. KYLX15_0940).

\section{References}

[1] Du M, Wang W, Jin H, et al. The association analysis of lncRNA HOTAIR genetic variants and gastric cancer risk in a Chinese population. Oncotarget, 2015;6:31255.

[2] Liang D, Liang S, Jin J, et al. Gastric cancer burden of last 40 years in North China (Hebei Province): A population-based study. Medicine (Baltimore), 2017;96:e5887.

[3] Zhao W, Zhu H, Zhang S, et al. Trop2 is overexpressed in gastric cancer and predicts poor prognosis. Oncotarget, 2016;7:6136.

[4] Zhou ML, Wang L, Wang JZ, et al. Validation of the memorial sloan kettering cancer center nomogram to predict disease-specific survival in a chinese gastric cancer population receiving postoperative chemoradiotherapy after an R0 resection. Oncotarget, 2016;7:64757.
[5] Lv ZD, Zhao WJ, Jin LY, et al. Blocking TGF-betal by P17 peptides attenuates gastric cancer cell induced peritoneal fibrosis and prevents peritoneal dissemination in vitro and in vivo. Biomed Pharmacother, 2017;88:27.

[6] Lin JC, Wu YY, Wu JY, et al. TROP2 is epigenetically inactivated and modulates IGF-1R signalling in lung adenocarcinoma. Embo Mol Med, 2012;4:472.

[7] Cubas R, Li M, Chen C, et al. Trop2: a possible therapeutic target for late stage epithelial carcinomas. Biochim Biophys Acta, 2009;1796:309.

[8] Wanger TM, Dewitt S, Collins A, et al. Differential regulation of TROP2 release by $\mathrm{PKC}$ isoforms through vesicles and ADAM17. Cell Signal, 2015;27:1325.

[9] Lin H, Huang JF, Qiu JR, et al. Significantly upregulated TACSTD2 and Cyclin D1 correlate with poor prognosis of invasive ductal breast cancer. Exp Mol Pathol, 2013;94:73.

[10] Wang H, Liu QQ, Tang XJ, et al. Eukaryotic expression of human anti-TROP2 antibody IgG, its inhibitory effect on cell proliferation of pancreatic cancer. Acta Univ Med Nanjing, 2014;34(7):863-9.

[11] Lin H, Zhang H, Wang J, et al. A novel human Fab antibody for Trop2 inhibits breast cancer growth in vitro and in vivo. Int J Cancer, 2014;134:1239.

[12] $\mathrm{Hu} \mathrm{L}$, Wang $\mathrm{H}$, Zhao $\mathrm{Y}$, et al. 125I seeds radiation induces paraptosis-like cell death via PI3K/AKT signaling pathway in HCT116 cells. Biomed Res Int, 2016;2016:8145495.

[13] Sun J, Wang H, Liu B, et al. Rutin attenuates $\mathrm{H}_{2} \mathrm{O}_{2}-$ induced oxidation damage and apoptosis in Leydig cells by activating PI3K/Akt signal pathways. Biomed Pharmacother, 2017;88:500.

[14] Liu C, Liang X, Wang J, et al. Protein O-fucosyltransferase 1 promotes trophoblast cell proliferation through activation of MAPK and PI3K/Akt signaling pathways. Biomed Pharmacother, 2017;88:95.

[15] Feng X, Wu C, Yang M, et al. Role of PI3K/Akt signal pathway on proliferation of mesangial cell induced by HMGB1. Tissue Cell, 2016;48:121. 
[16] Ejeskar K, Vickes O, Kuchipudi A, et al. The unique non-catalytic C-terminus of P37delta-PI3K adds proliferative properties in vitro and in vivo. PLoS One, 2015;10:e127497.

[17] Cui H, Seubert B, Stahl E, et al. Tissue inhibitor of metalloproteinases-1 induces a pro-tumourigenic increase of miR-210 in lung adenocarcinoma cells and their exosomes. Oncogene, 2015;34:3640.

[18] Dan HC, Antonia RJ, Baldwin AS. PI3K/Akt promotes feedforward mTORC2 activation through IKKalpha. Oncotarget, 2016;7:21064.

[19] Lu Y, Li Y, Chai X, et al. Long noncoding RNA HULC promotes cell proliferation by regulating PI3K/AKT signaling pathway in chronic myeloid leukemia. Gene, $2017 ; 607: 41-6$

[20] Zhao W, Zhu H, Zhang S, et al. Trop2 is overexpressed in gastric cancer and predicts poor prognosis. Oncotarget, 2016;7:6136.

[21] Sukhthankar M, Alberti S, Baek SJ. (-)-Epigallocatechin-3-gallate (EGCG) post-transcriptionally and posttranslationally suppresses the cell proliferative protein TROP2 in human colorectal cancer cells. Anticancer Res, 2010;30:2497.

[22] Guerra E, Trerotola M, Dell' AR, et al. A bicistronic CYCLIN D1-TROP2 mRNA chimera demonstrates a novel oncogenic mechanism in human cancer. Cancer Res, 2008;68:8113.

[23] Cubas R, Zhang S, Li M, et al. Chimeric Trop2 viruslike particles: a potential immunotherapeutic approach against pancreatic cancer. J Immunother, 2011;34:251.

[24] Liu T, Tian J, Chen Z, et al. Anti-TROP2 conjugated hollow gold nanospheres as a novel nanostructure for targeted photothermal destruction of cervical cancer cells. Nanotechnology, 2014;25:345103.
[25] Wang J, Zhang K, Grabowska D, et al. Loss of Trop2 promotes carcinogenesis and features of epithelial to mesenchymal transition in squamous cell carcinoma. Mol Cancer Res, 2011;9:1686.

[26] Gao XY, Zhu YH, Zhang LX, et al. siRNA targeting of Trop2 suppresses the proliferation and invasion of lung adenocarcinoma H460 cells. Exp Ther Med, 2015;10:429.

[27] Goldstein AS, Lawson DA, Cheng D, et al. Trop2 identifies a subpopulation of murine and human prostate basal cells with stem cell characteristics. Proc Natl Acad Sci U S A, 2008;105:20882.

[28] Chen MB, Wu HF, Zhan Y, et al. Prognostic value of TROP2 expression in patients with gallbladder cancer. Tumour Biol, 2014;35:11565.

[29] Pak MG, Shin DH, Lee CH, et al. Significance of EpCAM and TROP2 expression in non-small cell lung cancer. World J Surg Oncol, 2012;10:53.

[30] Shvartsur A, Bonavida B. Trop2 and its overexpression in cancers: regulation and clinical/therapeutic implications. Genes Cancer, 2015;6:84.

[31] Cubas R, Zhang S, Li M, et al. Trop2 expression contributes to tumor pathogenesis by activating the ERK MAPK pathway. Mol Cancer, 2010;9:253.

[32] Vidmar T, Pavsic M, Lenarcic B. Biochemical and preliminary $\mathrm{X}$-ray characterization of the tumor-associated calcium signal transducer 2 (Trop2) ectodomain. Protein Expr Purif, 2013;91:69.

[33] Stoyanova T, Goldstein AS, Cai H, et al. Regulated proteolysis of Trop2 drives epithelial hyperplasia and stem cell self-renewal via beta-catenin signaling. Genes Dev, 2012;26:2271.

(Received 21 April 2017, Revised 02 May 2017, Accepted 14 May 2017) 